\title{
The impact of delirium on clinical outcomes in multi-center Thai Surgical Intensive Care Units: A prospective cohort study
}

Pipanmekaporn T, ${ }^{1}$ Chittawatanarat K, ${ }^{2}$ Chaiwat A, ${ }^{3}$ Thawitsri T, ${ }^{4}$ Kongsayreepong $S,{ }^{3} \quad$ Thai SICU Study Group

${ }_{1}$ Department of Anesthesiology, Faculty of Medicine, Chiang Mai University, Chiang Mai, 50200, Thailand, 2 Department of Surgery, Faculty of Medicine, Chiang Mai University,50200, Thailand, ${ }^{3}$ Department of Anesthesiology, Faculty of Medicine, Siriraj Hospital, Mahidol University,Bangkok, 10700,

Thailand, ${ }^{4}$ Department of Anesthesiology, Faculty of Medicine, King Chulalongkorn Memorial Hospital, Bangkok, 10330, Thailand

\section{Abstract}

Background and Goal of Study:The incidences of delirium in the intensive care unit among studies vary from $10 \%$ to $70 \%$ according to patient settings and tools for diagnosis of delirium. ${ }^{1}$ Delirium in the intensive care unit (ICU) increases the risk of prolonged mechanical ventilation, prolonged hospitalization, and mortality. ${ }^{2}$ The purpose of the study was to determine if delirium in the surgical intensive care units (SICU) was an independent risk factor of clinical outcomes during hospitalization.

Materials and Methods: A muti-center, prospective cohort study was conducted between April 2011 and January 2012. All patients who were admitted into nine university-based SICU were enrolled. Delirium was diagosed by using Delirium Screening Checklists. The short term clinical outcomes of the study included ICU mortality and 28-day hospital mortality. Cox proportional hazard multivariable regression analyses were used to assess the effects of delirium on its clincal outcomes and presented with hazard ratio (HR) and 95\% confidence interval (CI).Time to event was assessed using Kaplan Meier curve and compared using log-rank test.

Results and Discussion: A total of 4,652 patients were included. One hundred and sixty three patients were dignosed delirium at some points during the ICU stays $(3.5 \%, 163$ out of 4,652). Patients who experienced delirium were significantly older $(65.0 \pm 15.8$ years versus $61.6 \pm 17.3$ years, $p=$ 0.013), and had higher ASA PS (24.3\% versus 12.2, p < 0.001), higher Acute Physiology and Chronic Health Evaulation II score (APACHE II score) [16 (12-22) versus 10 (7-15), $\mathrm{p}<0.001$ ], and higher Sequential Organ Failure Assessment (SOFA) [5 (2-8) versus 2 (1-5), $p<0.001]$ compared to non delirium. Delirious patients also required longer ventilator days [7 (4-17) versus 2(1-4) days, $\mathrm{p}<0.001$ ], longer length of hospital stay [22 (14-34) versus 15 (9-26) days, $\mathrm{p}<0.001$ ], higher ICU mortality ( $24 \%$ versus $9.1 \%, \mathrm{p}<0.001)$, and higher 28 -day mortality $(28.2 \%$ versus $13.3 \%$, p < 0.001$)$ (Table 1). Delieirous patients were associated with increased ICU mortality (adjusted HR $=3.46$; 95\% CI: 1.19-10.04, $p=0.022$ ) and increased $28-$ day mortality (adjusted HR $=2.93 ; 95 \% \mathrm{CI}: 1.35-6.33, \mathrm{p}=0.006$ ) (Table 2). Figure 1 shows Kaplan Meier curve of survival upto 28 days after admission into hospital of both groups. The finding of this study was consistent with previous studies. ${ }^{2-3}$

Table 1 Patients characteristics

\begin{tabular}{|c|c|c|c|}
\hline \multicolumn{1}{|c|}{ Variables } & $\begin{array}{c}\text { Delirium } \\
(\mathbf{n = 1 6 3})\end{array}$ & $\begin{array}{c}\text { Non delirium } \\
(\mathbf{n = 4 , 4 8 9 )}\end{array}$ & P value \\
\hline Age (year) mean \pm SD & $65.5 \pm 15.8$ & $61.6 \pm 17.3$ & 0.013 \\
\hline Male (n, \%) & $103(63.2)$ & $2,626(58.5)$ & 0.232 \\
\hline $\begin{array}{l}\text { Body Mass Index }\left(\mathrm{kg} / \mathrm{m}^{2}\right) \\
\text { mean } \pm \text { SD }\end{array}$ & $22.0 \pm 4.6$ & $23.0 \pm 5.7$ & 0.027 \\
\hline ASA PS (n, \%) & & & $<0.001$ \\
\hline I-II & $26(15.9)$ & $1,340(29.8)$ & \\
\hline III & $58(35.6)$ & $1,690(37.6)$ & \\
\hline IV-VI & $27(16.6)$ & $422(9.4)$ & \\
\hline Comorbidity (n, \%) & & & \\
\hline Hypertension & $75(46.0)$ & $2,193(48.8)$ & 0.476 \\
\hline Diabetes mellitus & $33(20.2)$ & $985(22.0)$ & 0.607 \\
\hline CAD & $15(9.2)$ & $445(10.0)$ & 0.765 \\
\hline COPD & $21(12.9)$ & $191(4.3)$ & $<0.001$ \\
\hline CRF & $25(15.3)$ & $417(9.3)$ & 0.010 \\
\hline Emergency surgery (n, \%) & $80(66.7)$ & $1,068(30.3)$ & $<0.001$ \\
\hline $\begin{array}{l}\text { APACHE II Median } \\
\text { (P25-75) }\end{array}$ & $16(12-23)$ & $10(7-15)$ & $<0.001$ \\
\hline SOFA Median (P25-75) & $5(2-8)$ & $2(1-5)$ & $<0.001$ \\
\hline Ventilator days (days) & $7(4-17)$ & $779(18.2)$ & $<0.001$ \\
\hline $\begin{array}{l}\text { Length of ICU stay (days) } \\
\text { Median (P25-75) }\end{array}$ & $8(5-19)$ & $2(1-4)$ & $<0.001$ \\
\hline $\begin{array}{l}\text { Length of hospital stay } \\
\text { (days) Median (P25-75) }\end{array}$ & $22(14-34)$ & $15(9-26)$ & $<0.001$ \\
\hline & & & \\
\hline
\end{tabular}

ASA PS; American Society of Anesthesiologists physical status, COPD; chronic obstructive pulmonary disease, APACHE II; Acute Physiology and Chronic Health Evaluation II, SOFA; Sequential Organ Failure Assessment.

Table 2. Delirium status and clinical outcome including ICU mortality and 28 -day mortality

\begin{tabular}{|c|c|c|c|}
\hline & Non delirium & Delirium & P value \\
\hline ICU mortality & & & \\
\hline Number (n, \%) & $408(9.1)$ & $39(24)$ & $<0.001$ \\
\hline Adjusted HR (95\% CI) * & Reference & $3.46(1.19-10.04)$ & 0.022 \\
\hline 28- day mortality & & & \\
\hline Number (n, \%) & $596(13.3)$ & $46(28.2)$ & $<0.001$ \\
\hline Adjusted HR (95\% CI)* & Reference & $2.93(1.35-6.33)$ & 0.006 \\
\hline
\end{tabular}

\section{Figure 1. Kaplan Meier analysis of delirium in 28 day mortality}

\section{Conclusion}

Delirium in ICU was a major predictor of ICU mortality and 28 day hospital mortality . Early detection of delirium in the intensive care unit and treat the modifiable risk factors may improve chance of survival.

\section{Acknowledgement}

This study was supported through funding by Chiang Mai University, the Royal College of Anesthesiology of Thailand, and National Research Council of Thailand.

\section{References:}

1. Ouimet S, Kavanagh BP, Gottfried SB, Skrobik Y. Incidence, risk factors and consequences of ICU delirium. Intensive Care Med. 2007;33:66-73. Ely EW, Shintani A, Truman B, et al. Delirium as a predictor of mortality in mechanically ventilated patients in the intensive care unit. JAMA. 2004; 14:1753-62.

2. Lin SM, Liu CY, Wang CH, et al. The impact of delirium on survival of mechanically ventilated patients. Crit Care Med. 2004; 32: 22549.

3. Lin SM, Liu CY, Wang CH, et al. The impact of delirium on survival of mechanically ventilated patients. Crit Care Med. 2004; 32: 22549. 